\title{
Genetic polymorphisms and retinal vein occlusion in an Italian population
}

\author{
L. De Polo ${ }^{1 *}$, P.E. Maltese ${ }^{2 *}$, E. Rigoni ${ }^{2}$, M. Bertelli², S. Cecchin ${ }^{2}$, G. Staurenghi ${ }^{1}$ \\ and G. Stoppa ${ }^{3}$ \\ 1Department of Ophthalmology, Luigi Sacco University Hospital, Milan, Italy \\ ${ }^{2}$ MAGI non-profit Human Medical Genetics Institute, Rovereto, Italy \\ ${ }^{3}$ Faculty of Economics, University of Trento, Trento, Italy \\ *These authors contributed equally to this study. \\ Corresponding author: P.E. Maltese \\ E-mail: paolo.maltese@assomagi.org \\ Genet. Mol. Res. 14 (4): 13337-13341 (2015) \\ Received May 2, 2015 \\ Accepted August 1, 2015 \\ Published October 26, 2015 \\ DOI http://dx.doi.org/10.4238/2015.October.26.30
}

ABSTRACT. In this study, we assessed the prevalence of polymorphisms in genes involved in hyperhomocysteinemia or hemostasis to shed light on their role, if any, in retinal vein occlusion (RVO). We recruited 37 Italian patients (17 men and 20 women) with a diagnosis of central or branch RVO based on fundus examination and retinal fluorescein angiography, as well as 45 healthy controls. Risk factors and family history of RVO of all subjects were recorded. The distributions of polymorphisms in patients and controls were evaluated using the $X^{2}$ test and OR. We confirmed an increased risk in subjects with dyslipidemia (high density lipoprotein $<59 \mathrm{mg} / \mathrm{dL}$ : $17.8 \%$ of controls, $43.2 \%$ of patients, $P=0.0002$; low density lipoprotein $>130 \mathrm{mg} / \mathrm{dL}: 26.7 \%$ controls, $54.1 \%$ patients, $\mathrm{P}=0.0002)$, arterial hypertension $(60 \%$ controls, $75.7 \%$ patients, $\mathrm{P}$ $=0.023)$, and high body mass index $(28.9 \%$ controls, $70.3 \%$ patients, $P<$ 0.0001 , and excluded involvement of the selected polymorphisms in RVO. Overall, the tested polymorphisms did not appear to be useful for assessing predisposition or for the diagnosis and prognosis of RVO.

Key words: Retinal vein occlusion; RVO; Polymorphism; Thrombophilia 


\section{INTRODUCTION}

Retinal vein occlusion (RVO) is one of the most common retinal vascular diseases. It presents with sudden loss of vision and in severe cases, the damage caused by the thrombotic event might impair eyesight. Its pathogenesis is complex and many studies are underway to highlight predisposing risk factors. For some of these factors including hypertension, hyperlipidemia (O'Mahoney et al., 2008), and to a lesser extent diabetes mellitus, a link with the thrombotic event is well documented, whereas hyperhomocysteinemia is the most common "emerging" risk factor (Turello et al., 2010). Smoking is also significantly related to RVO (Turello et al., 2010) and body mass index (BMI) is a risk factor for branch RVO (EDCC Study Group, 1993).

Several studies in the literature have focused on the association between RVO and gene polymorphisms involved in hyperhomocysteinemia or hemostasis, but the results are inconsistent and the roles of the polymorphisms are not completely understood. These studies were primarily focused on the factor V Leiden polymorphism FVR506Q, the prothrombin (FII) G20210A variant, the C677T polymorphism of the methylene-tetra-hydro-folate-reductase (MTHFR) gene, 844ins68 in cystathionine b-synthase (CBS), ins287del in the angiotensin-converting enzyme (ACE) gene, and T235M in angiotensinogen ( $A G T)$ itself. We therefore performed a case-control study in which we evaluated both physiologic and gene polymorphism-based factors that could plausibly be predictive of RVO in the Italian population.

\section{MATERIAL AND METHODS}

In this study, we examined 37 randomly selected patients who had suffered a first episode of RVO (17 men and 20 women; aged $74.5 \pm 8.8$ years) recruited from the Eye Clinic of the University Hospital "Luigi Sacco", Milan, Italy. Twelve patients had central RVO and 25 had branch RVO. The diagnosis of RVO was based on fundus examination and retinal fluorescein angiography. All subjects signed informed consent to the genetic and to use of their clinical and genetic data for publication and research. Guidelines from the ethics committee of reference for the present study stated that if anonymized or in aggregate, diagnostic data bearing patient consent for research, does not require approval by the ethics committee because the data cannot be traced back to the persons involved, even indirectly. The control population consisted of 45 healthy volunteers (22 men and 23 women; aged $78.6 \pm 9.2$ years) with no family history of RVO, selected from a collection of DNA samples from donors recruited for research studies. In both populations, we analyzed systemic and "emerging" factors such as arterial hypertension (HTN), dyslipidemia (low (LDL) and high (HDL) density lipoproteins and triglycerides), diabetes (defined as glycosylated hemoglobin $\mathrm{A} 1 \mathrm{C}$ or $\mathrm{HbA} 1 \mathrm{C}>6.5 \%$ ), smoking, $\mathrm{BMI}$ (weight in kilograms divided by height in meters squared, $\mathrm{kg} / \mathrm{m}^{2}$ ), and mutations in the genes for factor $V$ Leiden (G1691A mutation), FII (G20210A mutation), MTHFR (C677T mutation), CBS (844ins68 mutation), ACE (IVS16ins287 mutation), and AGT (T803C or Met268Thr also known as M235T).

DNA was extracted from $0.5 \mathrm{~mL}$ whole blood using a kit based on the salting-out method (Blood DNA kit E.N.Z.A., Omega bio-tek; Norcross, GA, USA).

Each 25- $\mu \mathrm{L}$ PCR reaction mixture contained 30-50 ng DNA, $10 \mathrm{pmol} / \mu \mathrm{L}$ each primer, 2.5 $\mu \mathrm{L}$ 10X PCR buffer, $0.5 \mathrm{mM}$ each dNTP, $1.5 \mathrm{mM} \mathrm{MgCl}_{2}$, and $0.75 \mathrm{U}$ Taq polymerase (Fermentas; Vilnius, Lithuania). The reactions were carried out in a thermocycler (Esco; Hamburg, Germany). After amplification, $3 \mu \mathrm{L}$ amplification product was subjected to electrophoresis on a $2 \%$ agarose gel stained with Gel Red (Biotium; Hayward, CA, USA) and observed under ultraviolet light. PCR 
product sizes were estimated by comparison with a 100-bp DNA ladder as a marker (Fermentas).

For the PCR, we used the following thermal profile: $95^{\circ} \mathrm{C} \times 5 \mathrm{~min}$ of initial denaturation, $95^{\circ}$ for $15 \mathrm{~s}$ for continuous denaturing, $30 \mathrm{~s}$ for annealing, $72^{\circ}$ for $40 \mathrm{~s}$ for polymerization, followed by a final extension at $72^{\circ}$ for $5 \mathrm{~min}$; annealing temperatures and number of cycles were specific for each primer pairs.

The DNA regions containing polymorphisms were amplified with the following primer pairs and specific PCR conditions: Factor $V$ Leiden (G1691A mutation) primer $F$ : TGCCCAGTGCTTAAC AAGACCA, primer R: CTTGAAGGAAATGCCCCATTA, annealing temperature $59^{\circ} \mathrm{C}$, 35 cycles; FII (G20210A mutation) primer F: TCTAGAAACAGTTGCCTGGC, primer R: ATAGCACTGGGAGC ATTGAAGC , annealing temperature $60^{\circ} \mathrm{C}, 35$ cycles; MTHFR (C677T mutation) primer F: TCG CCTTGAACAGGTGGAG, primer R: GACGGTGCGGTGAGAGTG, annealing temperature $58^{\circ} \mathrm{C}$, 35 cycles; CBS (844ins68 mutation) primer F: GCCACTCCCATAATAGAATA, primer R: TGTGAG GGTGAGTTACAGG, annealing temperature $58^{\circ} \mathrm{C}, 35$ cycles; ACE (IVS16ins 287 mutation) primer F: CTGGAGACCACTCCCATCCTTTCT, primer R: GACGTGGCCATCACATTCGTCAGAT, annealing temperature $63^{\circ} \mathrm{C}$, 35 cycles; AGT (T803C or Met268Thr also known as M235T) primer F: CTACAGGCAATCCTGGGTGT, primer R: TTGCCTTACCTTGGAAGTGG, annealing temperature $58^{\circ} \mathrm{C}, 37$ cycles.

Factor V Leiden, FII and MTHFR PCR products were digested with Mnll $10 \mathrm{u} / \mu \mathrm{L}$ (Fermentas) $1 \mathrm{~h}$ at $37^{\circ} \mathrm{C}$, Hind III $10 \mathrm{u} / \mu \mathrm{L}$ (Fermentas), $5 \mathrm{~min}$ at $37^{\circ} \mathrm{C}$ and Hinf1 $10 \mathrm{u} / \mu \mathrm{L}$ (Fermentas), $1 \mathrm{~h}$ at $37^{\circ} \mathrm{C}$, respectively and then separated on $2 \%$ agarose gel.

CBS (844ins68 mutation) were solved in a $2 \%$ agarose gel as follow: normal allele (wild type, N/N), 265bp; I = allele with insertion (homozygous mutation I/I), 333bp and heterozygous (N/I), 265/333; similarly, ACE (IVS16ins287 mutation) were solved in a $2 \%$ agarose gel as follow: allele with deletion (wild type, D/D), 191bp; I = allele with insertion (homozygous mutation I/I), 490bp and heterozygous (D/I), 191/490.

AGT PCR products were purified using the 'Cycle Pure Kit' (Omega bio-tek; Norcross, GA, USA) and sequenced using the same PCR amplification primers separately with the following thermal protocol: $96^{\circ} \mathrm{C}$ for $30 \mathrm{~s}$ denaturing, $52^{\circ} \mathrm{C}$ for $20 \mathrm{~s}$ annealing, and $60^{\circ} \mathrm{C}$ for 4 min extension repeated for 30 cycles using $6 \mu \mathrm{L}$ DCTS Quick Start Master Mix (GenomeLabTM DCTS-Quick Start Kit, Beckman Coulter), $5 \mu \mathrm{L}$ Sequencing Primer (1 pmol/uL) and $3.5 \mu \mathrm{L}$ amplified PCR product in a final volume of $20 \mu \mathrm{L}$. The product was purified again and suspended in $40 \mu \mathrm{L}$ Samlpe Loading Solution (GenomeLabTM DCTS-Quick Start Kit, Beckman Coulter) and analyzed in the Beckman Coulter CEQ 8000 automatic sequencer. The sequences produced by the samples were analyzed using BioEdit software (Hall, 1999).

For the $\mathrm{X}^{2}$ statistic, the factors BMI, HDL, LDL, and HTN were converted from quantitative results to categorical data by dividing the data into two groups: BMI risk class $(\mathrm{BMI} \geq 25)$ and no-risk class (BMI < 25) on the basis of the World Health Organization definition (pre-obese BMI: 25.00-29.99; obese BMI $\geq 30$ ); the HTN risk class (hypertensive) was defined as having systolic pressure of at least $140 \mathrm{mmHg}$ and/or diastolic pressure of at least $90 \mathrm{mmHg}$ while lower values defined the no-risk class (normotensive); cut-off values for HDL and LDL risk classes were $<59$ $\mathrm{mg} / \mathrm{dL}$ and $>130 \mathrm{mg} / \mathrm{dL}$, respectively. Statistical significance was set at $\mathrm{P}<0.05$ for the $X^{2}$ test and OR calculation (MedCalc software, Mariakerke, Belgium).

\section{RESULTS AND DISCUSSION}

Our results confirmed that the patient group had significantly lower blood levels of HDL 
cholesterol and higher LDL cholesterol than did the controls (low HDL: $8 / 45(17.8 \%)$ in controls, $16 / 37(43.2 \%)$ in patients, $P=0.0002$; high LDL: $12 / 45(26.7 \%)$ in controls, $20 / 37(54.1 \%)$ in patients, $\mathrm{P}=0.0002$ ) (O'Mahoney et al., 2008). Other factors such as arterial hypertension (27/45 $(60 \%)$ in controls, $28 / 37(75.7 \%)$ in patients, $\mathrm{P}=0.023)$ and high $\mathrm{BMI}(13 / 45(28.9 \%)$ in controls, $26 / 37(70.3 \%)$ in patients, $P<0.0001)$ were associated with a predisposition for the disease, in line with the literature (EDCC Study Group, 1993; O'Mahoney et al., 2008). In contrast, no significant association was found between RVO and diabetes $(P=0.085)$ or smoking $(P=0.06)$. Genetic evaluation did not show any differences in the distributions of the selected polymorphisms between patients and controls (Table 1), with the exception of FV G1691A ( $P=0.026$ ), which, however, failed to reach significance according to the OR.

Considering the small size of the two populations in our study and the limitations of retrospective study design, the results must be interpreted with caution, even if they are in line with those of the literature. Our results confirm that genetic determination of these polymorphisms is of no use for predicting a retinal thrombotic event (Weger et al., 2005; Baglin et al., 2010). In conclusion, genetic testing is not indicated in patients with RVO. Routine screening for these polymorphisms should not be recommended for determining prognosis or for decisions regarding treatment.

Table 1. Distribution of polymorphisms in patients and controls.

\begin{tabular}{|c|c|c|c|c|c|}
\hline Polymorphism & Genotypes & Patients & Controls & $P$ value* & OR (P value) \\
\hline \multirow[t]{3}{*}{ FV G1691A } & $\mathrm{G} / \mathrm{G}$ & $32(86 \%)$ & $43(96 \%)$ & 0.026 & Ref \\
\hline & $\mathrm{G} / \mathrm{A}$ & $5(14 \%)$ & $2(4 \%)$ & & $3.36(0.16)$ \\
\hline & $\mathrm{A} / \mathrm{A}$ & $0(0 \%)$ & $0(0 \%)$ & & - \\
\hline \multirow[t]{3}{*}{$F / l$ G20210A } & $\mathrm{G} / \mathrm{G}$ & $37(100 \%)$ & $45(100 \%)$ & & - \\
\hline & $\mathrm{G} / \mathrm{A}$ & $0(0 \%)$ & $0(0 \%)$ & - & - \\
\hline & $\mathrm{A} / \mathrm{A}$ & $0(0 \%)$ & $0(0 \%)$ & & - \\
\hline \multirow[t]{3}{*}{ MTHFR C677T } & $\mathrm{C} / \mathrm{C}$ & $14(38 \%)$ & $15(33 \%)$ & 0.76 & Ref \\
\hline & $\mathrm{C} / \mathrm{T}$ & $13(35 \%)$ & $17(38 \%)$ & & $0.82(0.7)$ \\
\hline & $\mathrm{T} / \mathrm{T}$ & $10(27 \%)$ & $13(29 \%)$ & & $0.67(0.48)$ \\
\hline \multirow[t]{3}{*}{ CBS 844ins68 } & $\mathrm{N} / \mathrm{N}$ & $32(86 \%)$ & $41(91 \%)$ & 0.1 & Ref \\
\hline & $\mathrm{N} / \mathrm{I}$ & $5(14 \%)$ & $3(7 \%)$ & & $2.13(0.32)$ \\
\hline & $1 / 1$ & $0(0 \%)$ & $1(2 \%)$ & & $3.83(0.41)$ \\
\hline \multirow[t]{3}{*}{ ACE IVS16ins287 } & $\mathrm{D} / \mathrm{D}$ & $6(16 \%)$ & $7(16 \%)$ & 0.98 & Ref \\
\hline & $\mathrm{D} / \mathrm{l}$ & $15(41 \%)$ & $18(40 \%)$ & & $0.97(0.96)$ \\
\hline & $1 / 1$ & $16(43 \%)$ & $20(44 \%)$ & & $0.93(0.91)$ \\
\hline \multirow[t]{3}{*}{ xi T803C } & $\mathrm{T} / \mathrm{T}$ & $10(27 \%)$ & $13(29 \%)$ & 0.94 & Ref \\
\hline & $\mathrm{C} / \mathrm{T}$ & $20(54 \%)$ & $24(53 \%)$ & & $1.08(0.87)$ \\
\hline & $\mathrm{C} / \mathrm{C}$ & $7(19 \%)$ & $8(18 \%)$ & & $1.13(0.84)$ \\
\hline
\end{tabular}

$\mathrm{N}=$ normal allele; $\mathrm{I}=$ allele with insertion; $\mathrm{D}=$ allele with deletion; ${ }^{*} \mathrm{P}$ value from $\mathrm{X}^{2}$ test.

\section{Conflicts of interest}

The authors declare no conflict of interest.

\section{ACKNOWLEDGMENTS}

We are grateful to Helen Ampt for revising the manuscript.

\section{REFERENCES}

Baglin T, Gray E, Greaves M, Hunt BJ, et al. (2010). Clinical guidelines for testing for heritable thrombophilia. Br. J. Haematol. 149: 209-220. 
Hall TA (1999). BioEdit: a user-friendly biological sequence alignment editor and analysis program for Windows 95/98/NT. Nucl. Acids Symp. Ser. 41: 95-98.

O'Mahoney PR, Wong DT and Ray JG (2008). Retinal vein occlusion and traditional risk factors for atherosclerosis. Arch. Ophthalmol. 126: 692-699.

The Eye Disease Case-Control Study Group (EDCC) (1993). Risk factors for branch retinal vein occlusion. Am. J. Ophthalmol. 116: 286-296.

Turello M, Pasca S, Daminato R, Dello Russo P, et al. (2010). Retinal vein occlusion: evaluation of "classic" and "emerging" risk factors and treatment. J. Thromb. Thrombolysis 29: 459-464.

Weger M, Renner W, Steinbrugger I, Cichocki L, et al. (2005). Role of thrombophilic gene polymorphisms in branch retinal vein occlusion. Ophthalmology 112: 1910-1915. 